\title{
EFFECT OF BLUE LIGHT EXPOSURE TO BRAIN WAVE PHYSIOLOGY RELATED TO COGNITIVE AND MOTORIC FUNCTION DURING NIGHT DRIVING SIMULATION
}

\author{
LULU LUSIANTI FITRI*, DANDRI ALY PURAWIJAYA and SUPRIJANTO \\ Biology, School of Life Sciences and Technology, Institut Teknologi Bandung \\ Instrumentation and Control, Faculty of Industrial Technology, Institut Teknologi Bandung \\ *E-mail: lfitri@sith.itb.ac.id
}

Accepted 7 May 2020, Published online 6 July 2020

\begin{abstract}
Safety issue and accident of land transportation needs to be concerned about. One of the main reasons of most driving accidents is drowsiness since it is associated with circadian rhythm, which is formed by blue light in solar day light. Studies reported that blue light controls circadian rhythm and prevent drowsiness. This research aims to assess the effect of artificial blue light exposure at night that is expected to prevent drowsiness and behavior changes related to night driving. Behavioral changes can be observed from cognitive response activities that are evaluated by physiologic changes of brainwaves. Seven male subjects were chosen that met the assigned criteria. Brainwaves data was collected from 21.00 to 22.30 using the Electroencephalograph (EEG) from F3 and F4 channels for cognitive function. Results of blue light exposure suggested: (1) temporary effect on cognitive function, (2) fluctuation of brainwave activity in one minute interval, (3) increase hemispheric synchronization in cognitive function, and (4) delay brain asymmetric pattern in cognitive function. We concluded that blue light exposure influences the function of cognitive functions and has potential effect to prevent drowsiness.
\end{abstract}

Key words: Blue light, electroencephalograph, brain wave, cognitive, motoric, circadian rhythm, driving simulator

\section{INTRODUCTION}

The total number of land transportation accidents in 2005 is 47 million accidents and 81 million accidents in 2009 (Indonesia National Transport Department, 2010). Human factor is known as one of the important factors of land traffic accidents in Indonesia, which was $72 \%$ in 2019 alone (Republika, 2019). This data implies that accident numbers keep increasing each year and the highest frequency of accident may occur during nighttime. According to Petridou and Mustaki (2000), factors that contribute to accident was short term factors which include drowsiness, alcohol intoxication, satiety, and distraction. Previous research done by Boyce et al. (2006) and Garbarino et al. (2014) stated that drowsiness contributed for $20-30 \%$ in total of driving accident percentage.

Drowsiness may affect neurobehavioral disruption such as inattention (Beanland et al., 2003), a decrease in cognitive performance judged

\footnotetext{
* To whom correspondence should be addressed.
}

from the decrease of decision-making ability during measuring safe distance in driving and controlling speed level (West et al., 1993) or affect the decrease of motoric response in stepping over the brake when perceiving abrupt stimulus (Haufe et al., 2014). Blue light is a light that has wavelength peak at $468 \mathrm{~nm}$ and could be one of the alternatives for drowsiness modulation strategy. Some related researches in usage of blue lights are: (a) the increase of brain activity related to cognition (Vandelwell et al., 2013), (b) its effect on circadian rhythm (Tosini et $a l ., 2016)$ and (c) objective effect to drowsiness with Electroencephalograph (EEG) instrument (Yang et al., 2019).

A research using the blue light measured by EEG showed increase in brain motoric function activities in short time (millisecond) (Vandewalle et al., 2013), while the adaptation or stabilization to light exposure in retina needs 7 to 8 minutes (Teikari, 2006). Therefore, there is a necessity to evaluate further in relation to retina's light adaptation duration. As a comparison, the research of blue light exposure in extended length (6 hours) to 
retinal cell culture could lead to phototoxicity effect (Arnault et al., 2013). Previous research shows that EEG recording of distracted drivers have characteristics of constant alpha wave and fluctuating mu wave (Lin et al., 2011), while EEG recording during attentive state on driving shows an increase of beta wave (Lachat et al., 2012). Alpha and $\mathrm{Mu}$ waves are also interesting to be observed because both brainwaves are manifested in the same frequency $(8-13 \mathrm{~Hz})$, but have a different purpose. Hence, the aim of this study was to assess the effect of blue light exposure in driving task to cognitive and motoric function using brainwaves as a physiological parameter.

\section{MATERIALS AND METHODS}

\section{Subject criteria}

Total subjects supported in this research were 7 males and they were all students of Institut Teknologi Bandung and were chosen with the following criteria: 18-22 years old, able to drive a car, licensed driver, not color blind, normal eyesight or corrected, healthy, slept 8 hours the night before, not on drugs or any medication, did not consume any caffeine 24-hour prior data collection, and voluntarily attempted to be a part of this research. Subjects that fulfilled all criteria was given informed consent verbally before data collection.

\section{Materials}

Two units of Philips GoLite Blue with wavelength $\sim 467 \mathrm{~nm}$ and possible maximum light intensity settings (8 lux; measured at eye level) were used. The lights were placed 1 meter in front of the driver on both left and right side of the screen with exposure angle at $45^{\circ}$. EEG used in this research was FlexComp Infinity 10-Channel (Thought Technology Ltd, Canada) with $256 \mathrm{~Hz}$ data sampling resolution. The processed channels, for both left and right brain hemisphere consecutilvely, were F3, F4 for cognition area. Both brain hemispheres were monitored to observe the difference between them. Driving simulator program used was SCANIA Truck Driving Simulator, integrated with Genius Speed Wheel 5 for the steering controller, and projected on LG LED 42 " Screen.

\section{Data collection protocol}

Data was collected at Medical Instrumentation Laboratory at Faculty of Industrial Engineering, Physics Engineering Department, Institut Teknologi Bandung. The experiments were performed at 21.00 to $22.30 \mathrm{GMT}+7$ and when subjects were present at 21.00, they were equipped with the EEG electrodes while asked for consent. The EEG set up was done simultaneously with stimulus habituation until 22.00. During habituation phase, orbital channels were observed visually to see the feedback on the system while the subjects open and close their eyes. Data collection started at 22.00 and finished at 22.30 .

The subjects were randomly allocated between 2 possible protocols, which differ with the order of lighting condition (blue light followed by in dark or in dark followed by blue light) to counterbalance the carryover effect of the previous condition. All subjects were only recorded once. The brainwave data recording was performed for 8 minutes each during dark condition and blue light condition, consecutively. All data were processed with MATLAB software integrated with EEGLAB plugin to remove the data baseline and filtered at $8-30 \mathrm{~Hz}$. The data was further processed with specific wavelength filter and artifact removal to obtain the alpha, mu and beta wave. The wave data were transformed with square root method and statistically analyzed with General Linear Model (GLM): Repeated Measures in SPSS with the data of 7 subjects pooled and presented as average of 7 data point.

\section{RESULTS AND DISCUSSION}

According to analysis of brainwave voltage, blue light exposure had significant effect $(p<0.05)$ on alpha and beta wave distribution on cognitive area channels (Figure 1). In dark condition, alpha voltage averages are always higher and significantly different $(\mathrm{p}<0.05)$ (Figure 1a). Alpha wave has a specific trait i.e. its emergence depends on the availability of light and the emergence of alpha wave is in inverse comparison with the presence of light (Woertz et al., 2004). In other words, the presence of light will suppress alpha wave and on reversed condition, alpha wave will increase during dark or minimum presence of light.

On the other hand, blue light exposure in 8 minutes increased beta wave voltage significantly $(p<0.05)$ (Figure $1 b)$, but not for alpha wave. This implies that 8 minute duration of blue light exposure has shown the effect as seen from the increase of beta wave. When compared to the study by Cajochen et al. (2005) using blue light exposure in 2 hours duration, this study presumed that ipRGC cells excitation are already in response to blue light within 8 minutes and thus improving wakefulness due to melatonin hormone suppression. As observed in Figure 1b, during the first 4 minutes, distribution of beta wave were higher than alpha wave but from minute 5 to 8 , alpha wave was higher than beta wave. This condition may indicate that during minute 5 to 8 , there was an emergence of fatigue 
condition. Schrauf et al. (2011) stated that exhaustion during driving would increase duration and frequency of alpha wave. Lal (2001) also stated that the decrease of beta wave and the increase of alpha wave simultaneously indicate of driver's exhaustion and declining state of alertness. It can be inferred that blue light exposure in this research can maintain alertness in general during the first 4 minutes of exposure as seen from higher beta wave average compared to alpha wave.

After further observation in Figure 1b, there was an up and down fluctuation pattern each minute on beta wave which presumed to ipRGC mode of response. According to Teikari (2006), ipRGC cells have distinct characteristics compared to other photoreceptor cells which are slow response latency and activated in long duration (30 seconds) during exposed to stimulus. Moreover, according to Benson (2007) ipRGC has slow response latency (around 1 minute) and long depolarization duration. Warren et al. (2006) revealed that ipRGC response to light exposure in extended duration showed a gradual decrease from response peak which implied for an adaptation process in retina to light exposure. Statistical test of blue light exposure to beta wave were proven significantly different $(p<0.05)$ at 2 nd and 5 th minute (Figure $1 \mathrm{~b}$ ), which implied blue light exposure caused variance of beta wave with lowest peak during 2nd minute and highest peak at 5 th minute. In 7 th and 8 th minutes, alpha and beta wave increased but not statistically different $(p>0.05)$. This result suggests that blue light exposure only effective for 5 minutes to all subjects. Compared to previous studies, this research result explains that ipRGC activation after blue light exposure occurred in 1 minute. At the next minute, the activity was decreased in response to hyperpolarization phase or the condition where a nerve system cannot perceive stimulus as mentioned by Teikari (2006). Activity pattern and hyperpolarization of ipRGC continued until 5th minute and after that, ipRGC would be adapted to stimulus so the pattern of brainwave response would not follow ipRGC activation pattern anymore. This fact is an opportunity for further research regarding to latency and duration of effective blue exposure.

After observing the effect of blue light exposure on alpha and beta wave in general, the next step was an observation on the effect of the exposure on voltage average distribution in F3 and F4 channels, which are channels related to cognitive area (Figure 2). In opposite of F3 and F4 channels voltage average distribution in dark condition (Figure 2a), blue light exposure was shown to increase synchronization of F3 and F4 channels that promoted similarity in pattern except on 7 th minute (Figure 2b). Blue light exposure increased voltage average significantly $(\mathrm{p}<0.05)$ to both F3 and F4 channels and synchronization of both channels indicated the symmetry processing of both channels until 7 th minute. During the 8 th minute, the dominance of F3 channel could be seen when compared to dark condition (Figure 2a), dominance of F3 channel already happened at 5th minute. This result implies that blue light exposure can promote the delay of left hemisphere dominance represented by F3 channel. In correlation with ipRGC adaptation hypothesis in Figure 1b, it can be assumed that the symmetry during 5 th to 7 th minutes is symmetry from hyperpolarization phase of ipRGC. Hyperpolarization phase on 5 th minute indicated ipRGC was on recovery phase to recover melanopsin into active state. This recovery phase occurred after 5 th minute until 8th minute so it can be presumed this phase happened in 3 minutes' duration. Moreover, blue light exposure is assumed not perceived anymore by ipRGC on 6th to 8th minute so the channels symmetry of cognitive area was prone to disappear. At this moment, there has not been a research related to frontal channels symmetry to psychological aspect manifestation. Although, Rusinov et al. (1976) stated that channels symmetry occurred in healthy subjects. Despite the fact, that 7 healthy subjects were used in this research, it

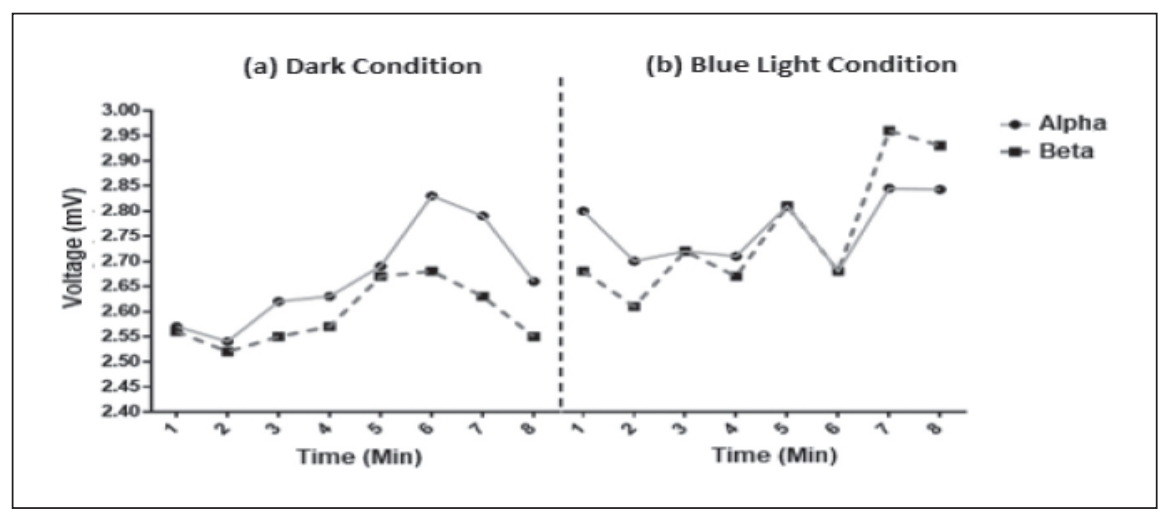

Fig. 1. Distribution of alpha and beta wave voltage average in cognitive area during 8 minutes in (a) dark condition and (b) blue light exposure condition. 


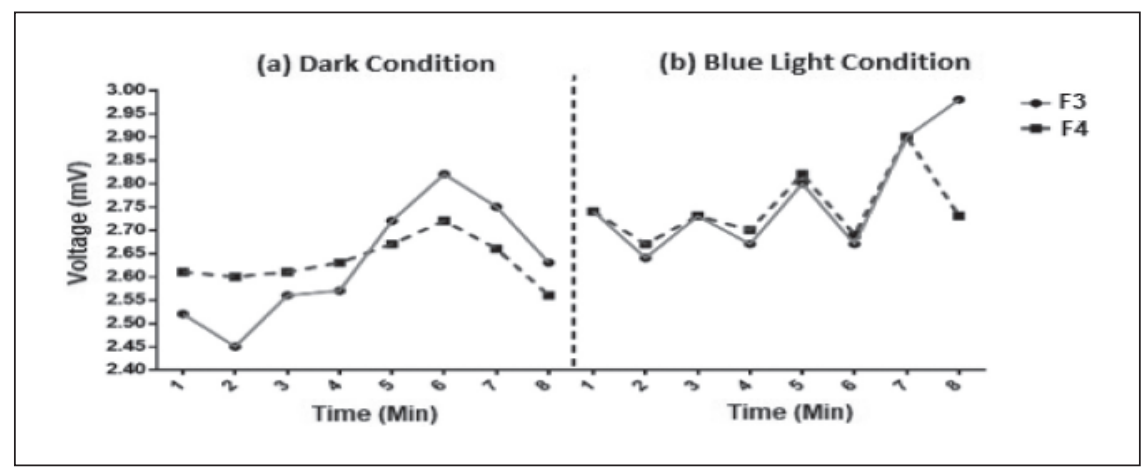

Fig. 2. Average voltage distributions of F3 and F4 channels in 8 minutes in (a) dark condition and (b) blue light exposure condition.

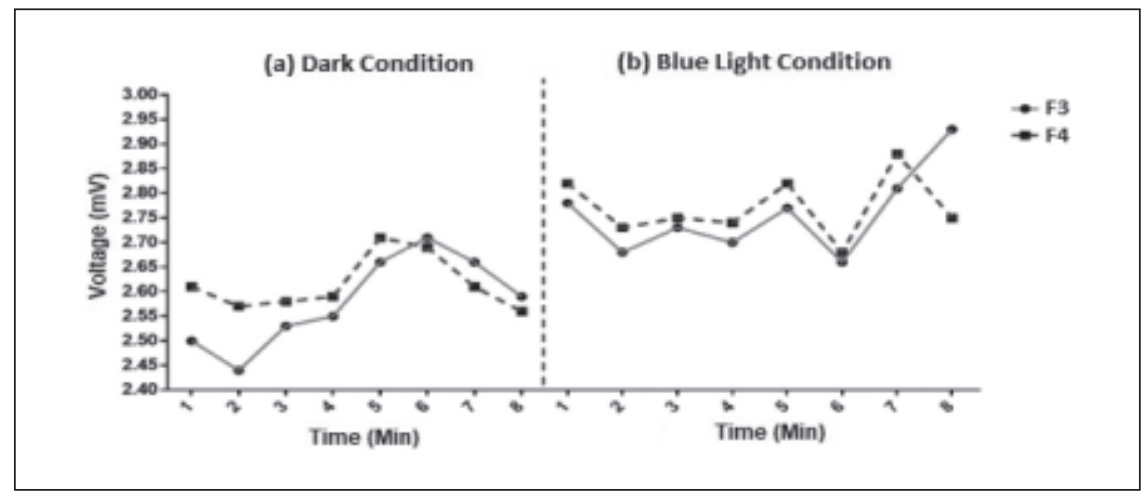

Fig. 3. Average voltage distribution of beta wave on F3 and F4 channels in 8 minutes in (a) dark condition and (b) blue light exposure condition.

indicates that blue light exposure can promote the symmetry of frontal channels has the potential to improve the driving performance based on healthiness of the subject.

Further observation was done to beta wave distribution on both F3 and F4 channels during dark condition (Figure 3a) and blue light exposure condition (Figure 3b). This beta distribution analysis was done to see focus level of the subjects using neurobehavioral approach. In dark condition, the exchange of dominance happened during the 6th minute, while during blue light exposure condition, dominance happened during the 8 th minute (Figure 3a). As observed in Figure 3b, blue light exposure increased the symmetry of channel F3 and F4. Other than that, blue light exposure also could delay the dominance of beta wave. According to Hoffman and Schutter (2012), dominance of beta wave on right hemisphere (represented by channel F4 in this research) correlated positively with aggressive emotion and promoting error in simple cognitive test. In relation with driver condition during driving, driving in the dark condition is not pleasant during the first 5 minutes because of F4 channel dominance (Figure 3a). During this unpleasant condition, it could promote error if there are any measurements, which imply the need for further study. The change of dominance in the 6th minute in dark condition (Figure 3a) implied the adaptation process and the decrease of aggressive behavior chance. On the other hand, blue light exposure prolonged the duration of beta wave dominance in right hemisphere of F4 channel (Figure $3 b$ ). Some possibilities concerning this prolonged dominance are that blue light exposure can promote aggressive behavior and giving harmful effect when exposed to driver in the nighttime. This possibility also implies the necessity for further study in relation to aggressive behavior and blue light exposure because the research by Hoffman and Schutter (2012) only measured aggressive behavior using questionnaire thus did not represent the exact aggressive behavior. This research indicate that blue light exposure has a significant effect $(p<0.05)$ to cognitive area of the brain and increase alpha and beta wave voltage mean in cognitive area for 5 minutes. Blue light exposure also promotes symmetry in left and right hemisphere of frontal lobe which indicates the increase of brain activity although one of possible neurobehavioral activity that promoted are aggressive behavior. Blue light exposure also indirectly increases arousal and 
indicates its ability to increase wakefulness during night driving.

Blue light is also presumed to be able to increase arousal in cognitive area, which could also increase the motoric input for the driver. Both arousal and motoric input will decrease when the driver is in drowsy situation. With blue light exposure that potentially could increase arousal and motoric input, blue light exposure indirectly has the potential to reduce the effect from drowsiness in driver during nighttime driving.

\section{CONCLUSION}

Blue light exposure can increase alpha and beta wave voltage in cognitive area and mu wave voltage in motoric area significantly with 5 mode of response: (a) blue light exposure is assumed only effective for 5 minutes in cognitive area, (b) the emergence of fluctuation pattern per minute in cognitive and motoric area, (c) increase in hemispheric synchronization on cognitive area, and (d) delay of dominance activity of left cognitive area (F3 channel). In conclusion, based on effect and response of blue light exposure to the increase of cognitive activity and ipRGC response, blue light exposure indirectly has the potential to decrease the effect of drowsiness.

\section{ACKNOWLEDGEMENT}

This research was supported by P3MI (Research, Community Service and Innovation Program) Institut Teknologi Bandung research funding year program 2017-2020.

\section{REFERENCES}

Arnault, E., Barrau, C., Nanteau, C., Gondouin, P., Bigot, K., Vienot, F., Gutman, E., Fontaine, V., Vilette, T., Cohen-Tannoudji, D., Sahel, J-A. \& Picaud, S. 2013. Phototoxic Action Spectrum on a Retinal Pigment Epithelium Model of AgeRelated Macular Degeneration Exposed to Sunlight Normalized Conditios. PloS One, 8(8): e71398.

Beanland, V.C., Fitzharris, M.P., Young, K.L. \& Lenne, M.G. 2013. Driver inattention and driver distraction in serious casualty crashes: data from the Australian National Crash In-depth Study. Accident Analysis and Prevention, 54: 99-107.
Benson, D.M. 2007. Phototransduction in ganglioncell receptors. European Journal of Physiology, 454: 849-855.

Boyce, P.R., Veitch, J.A., Newsham, G.R., Jones, C.C., Heerwagen, J., Myer, M. \& Hunter, C.M. 2006. Lighting quality and office work: two field simulation experiments. Lighting Research and Technology, 38(3): 191-233.

Cajochen, C., Munch, M., Kobialka, S., Krauchi, K., Steiner, R., Oelhafen, P., Orgul, S. \& WirzJustice, A. 2005. High Sensitivity of Human Melatonin, Alertness, Thermoregulation, and Heart Rate to Short Wavelength Light. Journal of Clinical Endocrinology and Metabolism, 90(3): 1311-1316

Garbarino, S., Gelsomino, G. \& Magnavita, N. 2014. Sleepiness, Safety and Transport. Journal of Ergonomy, S3: 003.

Haufe, S., Kim, J-W., Kim, I-H, Sonnleitner, A., Schrauf, M., Curio, G. \& Blankertz, B. 2014. Electrophysiology-based detection of emergency braking intention in real-world driving. Journal of Neural Engineering, 11(2014) 056011: 8.

Hofman, D. \& Schutter, D. 2012. Asymmetrical frontal resting-state beta oscillations predict trait aggressive tendencies and behavioral inhibition. SCAN, 7: 850-857.

Indonesia National Transport Department. 2010. Statistik Perhubungan Darat. [online] http:// gis.dephub.go.id/mapping/StatistikDarat.aspx on 4 April 2013.

Lachat, F., Hugueville, L., Lemarechal, J.D., Conty, L. \& George, N. 2012. Oscillatory brain correlates of live joint attention: a dual-EEG study. Frontiers in Human Neuroscience, 6(156): 1-12.

Lal, S. 2001. Behavioural Road Safety Management. [Online] http://acrs.org.au/files/arsrpe/ RS040009.pdf

Lin, C-T., Chen, S-A., Chiu, T-T., Lin, H-Z. \& Ko, L-W. 2011. Spatial and temporal EEG dynamics of dual-task driving performance. Journal of Neuroengineering and Rehabilitation, 8(11): 113.

Petridou, E. \& Moustaki, M. 2000. Human Factors in the causation of road traffic crashes. European Journal of Epidemiology, 16: 819826

Republika, 2019. [online] https://nasional. republika.co.id/berita/q37i3n423/jumlahkecelakaan-lalu-lintas-naik-3-persen-pada2019). 
Rusinov, V.S., Boldyreva, G.N. \& Vakar, E.M. 1976. Symmetry and stability of EEG spectra in healthy subjects. Neuroscience and Behavioural, 7(4): 348-357.

Schrauf, S., Simon, M., Schmidt, E. \& Kincses, W. 2011. Assessing Drivers' Fatigue State Under Real Traffic Conditions Using EEG Alpha Spindles. PROCEEDINGS of the Sixth International Driving Symposium on Human Factors in Driver Assessment, Training and Vehicle Design, Conference: Driving Assessment, Vol: 61-7.

Teikari. 2006. Biological Effects of Light. Master Thesis: Helsinki University of Technology.

Tosini, G., Ferguson, I. \& Tsubota, K. 2016. Effects of Blue Light on The Circadian System and Eye Physiology. Mollecular Vision, 22: 61-72.

Vandewalle, G., Collignon, O., Hull, J.T., Daneault, V., Albouy, G., Lepore, F., Philips, C., Doyon, J., Czeisler, C.A., Dumont, M., Lockley, S.W. \& Carrier, J. 2013. Blue light stimulates cognitive brain activity in visually blind individuals. Journal of Cognitive Neuroscience, 25(12): 2072-2085
Warren, E.J., Perez-Leon, J.A., Allen, C.N., Robinson, D.W. \& Brown, R.L. 2006. Synaptic inputs to retinal ganglion cells that set the circadian clock. Europera Journal of Neuroscience, 24(4): 1117-1123.

West, R.J., French, D., Kemp, R. \& Elander, J. 1993. Direct observation of driving, self reports of driver behaviour, and accident involvement. Ergonomics, 36(5): 557-567.

Woertz, M., Pfurtscheller, G. \& Klimesch, W. 2004. Alpha power dependent light stimulation: dynamics of event-related (de)synchronization in human electroencephalogram. Cognitive Brain Research, 20: 256 -260.

Yang, M.Q., Chen, Q.W., Zhu Y.Y., Zhou, Q., Geng, Y.G., Lu, C.C.,Wang, G.F. \& Yang, C.M. 2019. The effects of intermittent light during the evening on sleepiness, sleep electroencephalographic spectral power and performance the next morning. Lighting Research and Technology, 51(8): 1143-1158. 